\title{
Windows over a New Low Energy Axion
}

\section{Claudio Corianò ${ }^{1}$ and Nikos Irges $^{2}$}

\author{
${ }^{1}$ Dipartimento di Fisica, Università del Salento, and \\ INFN Sezione di Lecce, Via Arnesano 73100 Lecce, Italy \\ ${ }^{2}$ Department of Physics and Institute of Plasma Physics, \\ University of Crete, GR-710 03 Heraklion, Greece
}

\begin{abstract}
We outline some general features of possible extensions of the Standard Model that include anomalous $U(1)$ gauge symmetries, a certain number of axions and their mixings with the CP-odd Higgs sector. As previously shown, after the mixing one of the axions becomes a physical pseudoscalar (the axi-Higgs) that can take the role of a modified QCD axion. It can be driven to be very light by the same non-perturbative effects that are held responsible for the solution of the strong CPproblem. At the same time the axi-Higgs has a sizeable gauge interaction, which is not allowed to the Peccei-Quinn axion, possibly explaining the PVLAS results. We point out that the Wess-Zumino term, typical of these models, can be both interpreted as an anomaly inflow from higher dimensional theories (second window) but also as a result of partial decoupling of an extra Higgs sector (and of a fermion) that leaves behind an effective anomalous abelian theory (first window) in a broken Stückelberg phase. The possibility that the axi-Higgs can be heavy, of the order of the Higgs mass or larger, however, can't be excluded. The potentialities for the discovery of this particle and of anomaly effects in the neutral current sector at the LHC are briefly discussed in the context of a superstring inspired model (second window), but with results that remain valid also if any of the two possibilities is realized in Nature.
\end{abstract}




\section{Introduction.}

Extensions of the Standard Model (SM) incorporating a gauge structure with additional abelian $U(1)$ 's may be of particular relevance in the search for new neutral currents at the LHC and may have profound implications in an attempt to uncover the unique extension of the Standard Model that Nature selects. Abelian extensions are quite ubiquitous both in Grand Unified Theories and in effective string theories, and in general these interactions can be viewed as the low energy remnants of larger symmetries. As such, they deserve special attention. Extra abelian gauge symmetries imply, as we have just mentioned, new neutral interactions ( $Z^{\prime}$ gauge bosons) and corrections to the interactions of the SM electroweak gauge bosons that should be quite small, given the accuracy of the electroweak precision data from LEP.

In this work we intend to underline some simple consequences of the effective low energy theory that one obtains under certain assumptions which are, however, quite generic if some abelian interactions are present both at the electroweak scale and above it; this second scale, that we call $M$, can be quite remote from the latter. The appearance of these interactions and low energy structures, because of their generality, could be produced by different mechanisms, but they have a common low energy imprint: the presence of a pseudoscalar that undergoes mixing with the electroweak Higgs so to produce a low energy theory that is unitary, though non-renormalizable. This pseudoscalar, that in a first work has been called "the axi-Higgs" [1] plays a subtle role in guaranteeing the unitarity of the low energy extension and is characterized by a mass and interactions that are sensitive both to the QCD $\theta$-vacuum but also to additional CP-odd phases of possible scalar potentials generated at higher energy and that survive in the low-energy theory. In this work we briefly outline the arguments and highlight some of the results, leaving some of the details to a future work. Phenomenological discussions of alternative approaches in which the axion-gauge field interaction is modified respect to traditional axion models can be found in [2] [3].

\section{The first window with a simple model}

We start from a simple example. A working model that shares the main features discussed above such as a Stückelberg mass term, an anomalous fermion spectrum and axion-gauge field interactions is described by the classical lagrangean

$$
\begin{aligned}
\mathcal{L}_{0}= & \left|\left(\partial_{\mu}+i B_{\mu}\right) \phi\right|^{2}-\frac{1}{4} F_{A}^{2}-\frac{1}{4} F_{B}^{2}+\frac{1}{2}\left(\partial_{\mu} b+M_{1} B_{\mu}\right)^{2}-\lambda\left(|\phi|^{2}-\frac{v^{2}}{2}\right)^{2} \\
& +\bar{\psi} i \gamma^{\mu}\left[\partial_{\mu}+i e A_{\mu}+i \gamma^{5} B_{\mu}\right] \psi+\lambda_{1} \bar{\psi}_{L} \phi \psi_{R}+\text { h.c. }
\end{aligned}
$$


that we call the $A$ - $B$ model, with $A$ being vector-like and massless, while $B$ is made massive by a combination of the Stückelberg and the Higgs mechanisms. The tree-level mass $M_{1}$, also called "the Stückelberg mass" combines with the vev of the Higgs field to generate the total mass of the $B$ gauge boson which is $\sqrt{M_{1}^{2}+\left(g_{B} v\right)^{2}}$. The anomaly of the fermion is due to its (purely) axial coupling to $B$. The mixing of the field $b$ and the Higgs field takes place after spontaneous symmetry breaking and is triggered by the Higgs field. Probably the simplest way to look at this lagrangean is to consider it to be the low energy effective theory of a first breaking, driven by a much heavier Higgs $(\Theta$, charged under $\left.U(1)_{B}\right)$ at a higher scale, whose magnitude appears frozen at lower energy.

Notice that the presence of the Stückelberg scalar, that can be thought of as the (surviving) phase of $\Theta$, indicates that, before symmetry breaking of the light Higgs, the theory is already in a broken phase (the Stückelberg phase). This picture clearly does not give any special role to the Stückelberg scalar, other than being a CP-odd component of another Higgs field. In this specific interpretation of the Higgs-Stückelberg system the scale $M$ is directly related to the vev of the heavy Higgs, denoted by $V$. When also the light Higgs takes a vev $(v)$, we will call the corresponding symmetry phase the Higgs broken phase.

Both symmetry phases, in principle, could be anomalous, even if the original theory was assumed to be anomaly-free. In fact, together with the radial component of a heavier Higgs also some of the fermions may have been integrated out. The phase is then a simple example of an incomplete decoupling of some mother theory with a larger symmetry. Clearly both the low energy theory and the mother theory need to share a gauged $U(1)_{B}$ symmetry.

The left-over fermion, here denoted by $\psi$, makes up the light spectrum. In the absence of the Higgs field, the axion $b$ has a simple derivative coupling to the $B$ gauge boson, and it is a Goldstone mode. In the presence of the vev of a Higgs field, Higgs-axion mixing occurs and one linear combination becomes massless but is now physical. We call this a massless axi-Higgs. Mass corrections to the axi-Higgs can be generated by the introduction of phases in a given potential, whose origin, at this point, is left unspecified. We will comment upon this in the following. It is given by

$$
V_{P Q}=\lambda_{2}\left(\phi e^{-i q_{B} g_{B} \frac{b}{M_{1}}}\right)+\lambda_{3}\left(\phi e^{-i q_{B} g_{B} \frac{b}{M_{1}}}\right)^{2}+2 \lambda_{4}\left(\phi^{*} \phi\right)\left(\phi e^{-i q_{B} g_{B} \frac{b}{M_{1}}}\right)+\cdots \text { c.c. }
$$

with $\lambda_{2}, \lambda_{3}$ and $\lambda_{4}$ suitable parameters. The dots refer to other allowed terms, an example of which can be found in [1]. If we denote as $V_{P Q}$ the ordinary Higgs potential (which is phase-independent), $V_{P} Q$ introduces a periodic dependence similar to the breaking of the PQ symmetry by non-perturbative effects in the $\theta$-vacuum. Then the complete 
potential considered here can be equivalently written as

$$
V(H, b)=V_{P Q}+V_{P} \phi+V_{P}^{*} \phi
$$

and we require that its minima are located at

$$
\langle b\rangle=0 \quad\langle\phi\rangle=v,
$$

which can be achieved by a suitable choice of its free parameters. The CP-odd phases of the scalar sector can be rotated into the physical axi-Higgs $(\chi)$ and a Nambu-Goldstone boson $G$, giving

$$
\begin{aligned}
\phi_{2} & =\frac{1}{M_{B}}\left(-M_{1} \chi+q_{B} g_{B} v G\right) \\
b & =\frac{1}{M_{B}}\left(q_{B} g_{B} v \chi+M_{1} G\right) .
\end{aligned}
$$

The phase dependence of the new potential $V_{P} \not$ plays a key role in determining the actual mass of the physical axion. While the expressions of the two linear combinations (5) remain true also for a vanishing $V_{P} \not$, when it is present, there are some important mass corrections generated as well.

More precisely, for a non-vanishing $V_{\mathbb{P} \not}$ the scalar mass matrix has one zero eigenvalue corresponding to the Goldstone boson $G$ and a non-zero eigenvalue corresponding to a physical axion field $-\chi$ - with mass

$$
m_{\chi}^{2}=-\frac{1}{2} c_{\chi} v^{2}\left[1+\frac{q_{B}^{2} g_{B}^{2} v^{2}}{M_{1}^{2}}\right]=-\frac{1}{2} c_{\chi} v^{2} \frac{M_{B}^{2}}{M_{1}^{2}} .
$$

The mass of this state is positive if $c_{\chi}<0$, with

$$
c_{\chi}=4\left(\frac{\lambda_{2}}{v^{3}}+\frac{4 \lambda_{3}}{v^{2}}+\frac{2 \lambda_{4}}{v}\right) .
$$

The massless axi-Higgs is obtained by sending the parameters of $V_{P} \varnothing$ to zero, that is $c_{\chi} \rightarrow 0$. Notice that this parameter plays an important role in establishing the size of the mass of $\chi$, and encloses also the corrections to the standard Higgs potential induced by the phase dependent potential. Notice also that at this point we obtain different scenarios depending on the size of these parameters. In particular they could be generated nonperturbatively similarly to the case of the PQ axion, due to the presence of the instantons in the QCD $\theta$-vacuum [4] and in that case they would be naturally small [5]. A scenario that would guarantee a large vev $V$ for the heavy Higgs $\Theta$ while not allowing a phase to develop in the scalar potential, is one where the effective scalar potential originates from a supersymmetric D-term in the presence of a non-zero Fayet-Iliopoulos term. Notice that this possibility leaves open a (third) window towards a connection with the FroggattNielsen mechanism [6]. 


\subsection{Gauge interactions of the axi-Higgs}

The theory described above suffers from gauge anomalies, true or apparent, and there could be, at this stage, questions about its implementation at the level of effective field theories. Clearly, it is reasonable to expect that the main shortcoming of an effective anomalous theory is its non-renormalizability, while its unitarity should be preserved.

In the absence of a low-energy Higgs-axion mixing from a scalar potential (i.e. $V_{P} Q \rightarrow$ 0 ) and in the absence of Yukawa couplings (vanishing fermion masses) we can show unitarity of the model by a direct analysis with ease. In the presence of Yukawa couplings in a broken Higgs phase the same computation is slightly more involved but also goes through.

The tests can be performed by analyzing the cancelation of the gauge dependence of the gauge fixed action in a specific (non-unitary) gauge.

This can be more easily shown in the $R_{\xi}$ gauge, using the BRST symmetry of the full effective action and the validity of the corresponding Slavnov-Taylor identities. However, prior to gauge fixing and in the presence of a gauge field-axion mixing, the anomalous effective action, defined (only) as the classical action plus the anomalous triangle diagrams - in our case these are the $(A A B)$ and the $(B B B)$ vertices - can be rendered gauge invariant by suitable counterterms.

A wide body of literature on anomalies, in the past, has tried to answer this puzzling question. It has been proposed, just from a field theoretical ground, that an anomalous theory can be ameliorated by a suitable Wess-Zumino term, that introduces an axion. These arguments have been brought up (quite long ago) in theories in which a nondynamical $\theta$-term [7, 8] could improve the analysis of the effective theory.

The basic conclusion of these investigations was that a Wess-Zumino term does not "cancel" the anomaly (see the discussion in [9]), but ameliorates the behavior of the theory allowing a perturbative power counting. Also, the addition of non-local counterterms that do restore gauge invariance [10] are not compatible with an effective renormalizable theory. We are going to illustrate how this power counting goes.

\section{$2.2 \quad$ Restoring gauge independence}

The introduction (just on a field theoretical basis) of a WZ term, a dimension- 5 operator that spoils renormalizability, is the price to pay in order to be able to discuss the gaugeindependence of the S-matrix amplitudes of the model. This is illustrated in Fig. 1, where the diagrams are analyzed in the unitary gauge. Both diagrams are needed to remove the gauge dependence of the first diagram, the second one being purely gauge dependent. 


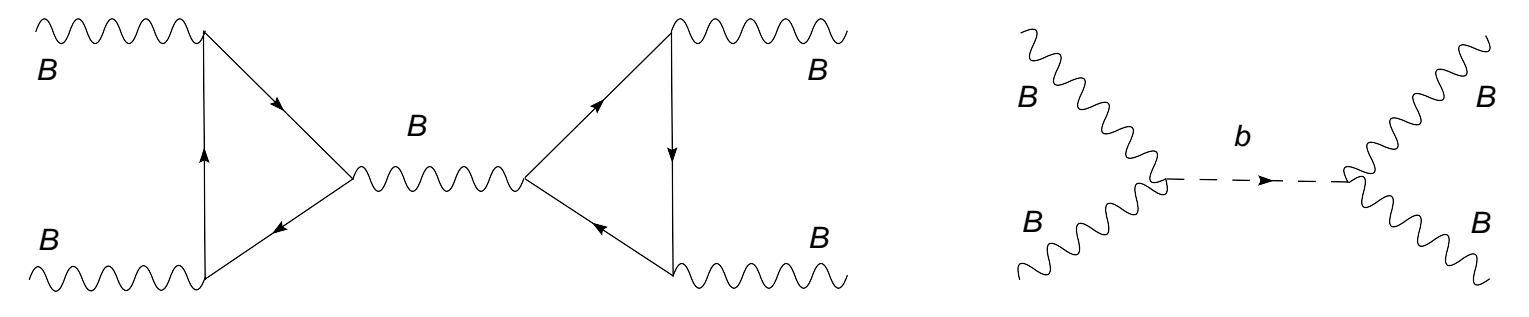

Figure 1: Diagrams relevant for the analysis of the gauge independence in the $R_{\xi}$ gauge for a B exchange.

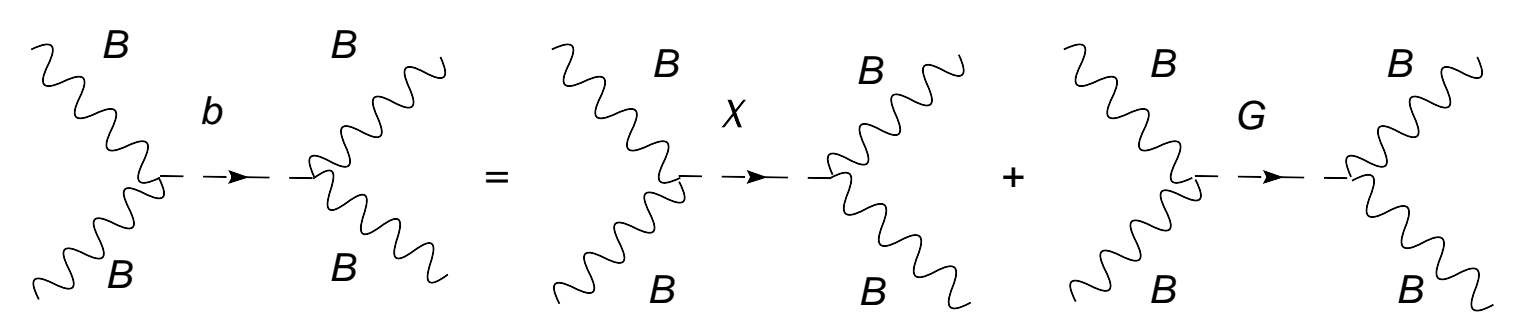

Figure 2: Projection of the axion b over the axi-Higgs and the Goldstone in the presence of Higgs-axion mixing.

Had we not introduced this term, we would be forced to cancel completely the gauge dependence of the first diagram just by going to the unitary gauge. In the unitary gauge $b$ is set to vanish and the second diagram disappears. In the presence of a Higgs, however, the same diagram does not disappear, since the unitary gauge choice does not eliminate the exchange of a physical axion, which corresponds to the exchange of a massless or massive pole depending on the absence/presence of a phase dependent potential. In this case the axion is rotated as in Fig. 2.

\section{$2.3 \quad$ Unitarity}

We are now going to show that the theory constructed out of the Stückelberg term and with/without a Yukawa coupling is unitary in the broken Higgs phase - the presence of the WZ term being necessary - by a direct computation.

The simplest approach to test a non-unitary behavior of a theory is to look for the presence of spurious s-channel poles in a variety of scattering amplitudes involving the anomalous diagrams of the model as in Bouchiat, Iliopoulos and Meyer [11. The proof is quite simple and can be identified by following the patterns of cancelations of the gauge dependence in s-channel exchanges such as those in Fig. 1.

Moving to the same analysis in the broken Higgs phase, a quick look at Fig. 3 may help in identifying the same pattern in the presence of Yukawa couplings. The exchange 


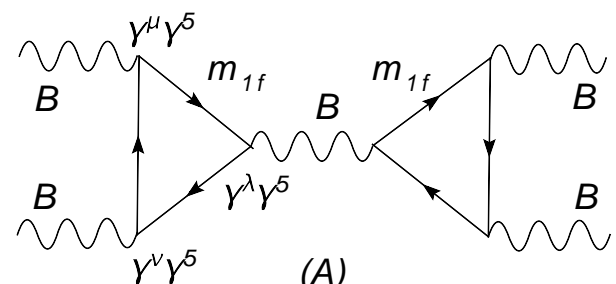

( $A)$

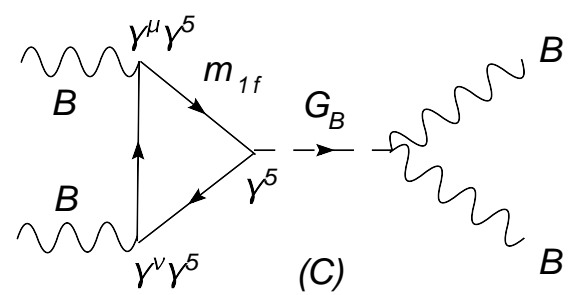

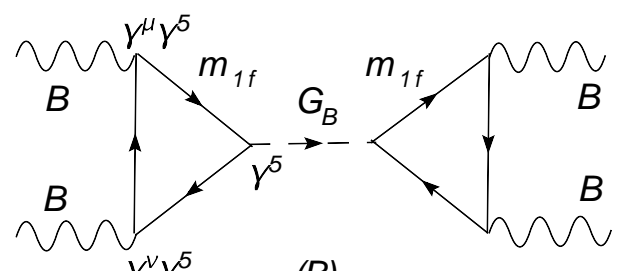

(B)

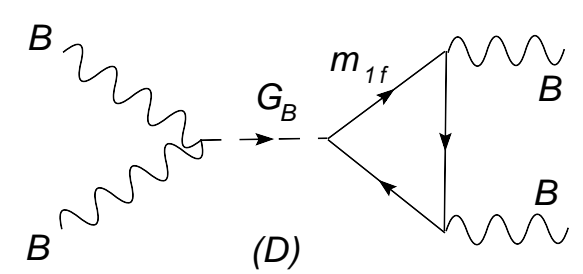

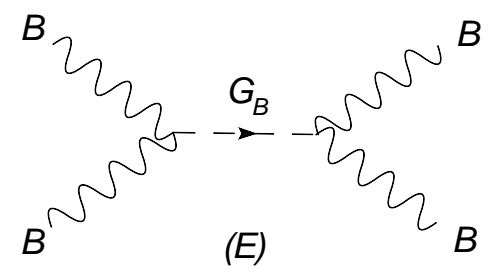

Figure 3: Gauge dependence cancelation after spontaneous symmetry breaking.

of the axion $b$ is rotated into the Goldstone and into the (gauge independent) physical axiHiggs (not included in Fig. 2). The pattern of cancelations then proceeds very similarly to the case of the SM and can be shown explicitly. We have not used any additional counter-term of Chern-Simons (CS) type to achieve this result, though, in principle, the theory may allow for terms of this type [1, 12]. In this case a Ward identity on the $A$ gauge boson determines the parametrization of the anomalous triangle diagram (which is defined modulo a momentum shift) so that the $A$-vector current is conserved. This condition, in fact, is a defining Ward identity on the model, corresponding to gauge invariance under $A$-gauge transformations. Similar Ward identities need to be satisfied if certain symmetries (residual gauge symmetries) are left over (such as $U(1)$ em gauge invariance) after symmetry breaking.

\subsection{The role of the Chern-Simons interactions}

We have seen, by a specific example, that the WZ term is more than just a gauge artifact in the low energy effective action and plays a considerable role in restoring the unitarity of the theory even in a physical gauge, since the axi-Higgs contributes to a given S-matrix element in a gauge independent way. Besides the WZ interaction, the model, in principle, allows direct gauge interactions mediated by Chern-Simons terms. While in more general models involving extensions of the SM that include more than a single anomalous $U(1)$ 
these interactions are part of the effective action, in the case of the model that we have illustrated this role is lost. It is however interesting to re-analyze the same pattern of cancelations in a more general case.

Therefore, we consider the following modification on the $A B$ model where the CS interactions are generically introduced as possible counterterms, together with WZ terms, in the 1-loop effective action, which is given by

$$
\mathcal{L}=\mathcal{L}_{0}+\mathcal{L}_{W Z}+\mathcal{L}_{C S}
$$

where $\mathcal{L}_{0}$ is the starting lagrangean, but in particular we focus on

$$
\mathcal{L}_{W Z}=\frac{C_{A A}}{M} b F_{A} \wedge F_{A}+\frac{C_{B B}}{M} b F_{B} \wedge F_{B},
$$

which corresponds to the WZ term (or Green-Schwarz in the string language) and on the term

$$
\mathcal{L}_{C S}=d_{1} B^{\mu} A^{\nu} F_{A}^{\rho \sigma} \epsilon_{\mu \nu \rho \sigma}=d_{1} B A F^{A}
$$

which denotes the gauge variant CS interaction, where gauge invariance fixes the unknown coefficients via the specific relations

$$
\begin{aligned}
C_{A A} & =\left(-\frac{d_{1}}{2}+\frac{i}{2 !} a_{3}(\beta) \frac{1}{4}\right) \frac{M}{M_{1}} \\
C_{B B} & =\frac{i}{3 !} \frac{a_{n}}{4} \frac{M}{M_{1}} .
\end{aligned}
$$

One important comment is in order. It is of considerable importance to observe that in these models the size of the interaction of the axi-Higgs to the gauge fields, $1 / M$, remains unrelated to the mechanism that generates its mass. This is an important variant compared to the standard PQ invisible axion, where they are both controlled by the same parameter $1 / f_{a}$ and both the mass term and the axion gauge interactions are equally suppressed by the same scale $\left(f_{a} \sim 10^{10} \mathrm{GeV}\right)$. It is by now well known that this point determines a disagreement between the PVLAS result [13] and its interpretation as a traditional axion [14].

In the equations above, $\beta$ is a shift parameter relevant for the distribution of the anomalies in the $A A B$ diagram, which in this case are given by

$$
\begin{aligned}
a_{1}(\beta) & =a_{2}(\beta)=-\frac{i}{8 \pi^{2}}-\frac{i}{4 \pi^{2}} \beta \\
a_{3}(\beta) & =-\frac{i}{4 \pi^{2}}+\frac{i}{2 \pi^{2}} \beta,
\end{aligned}
$$



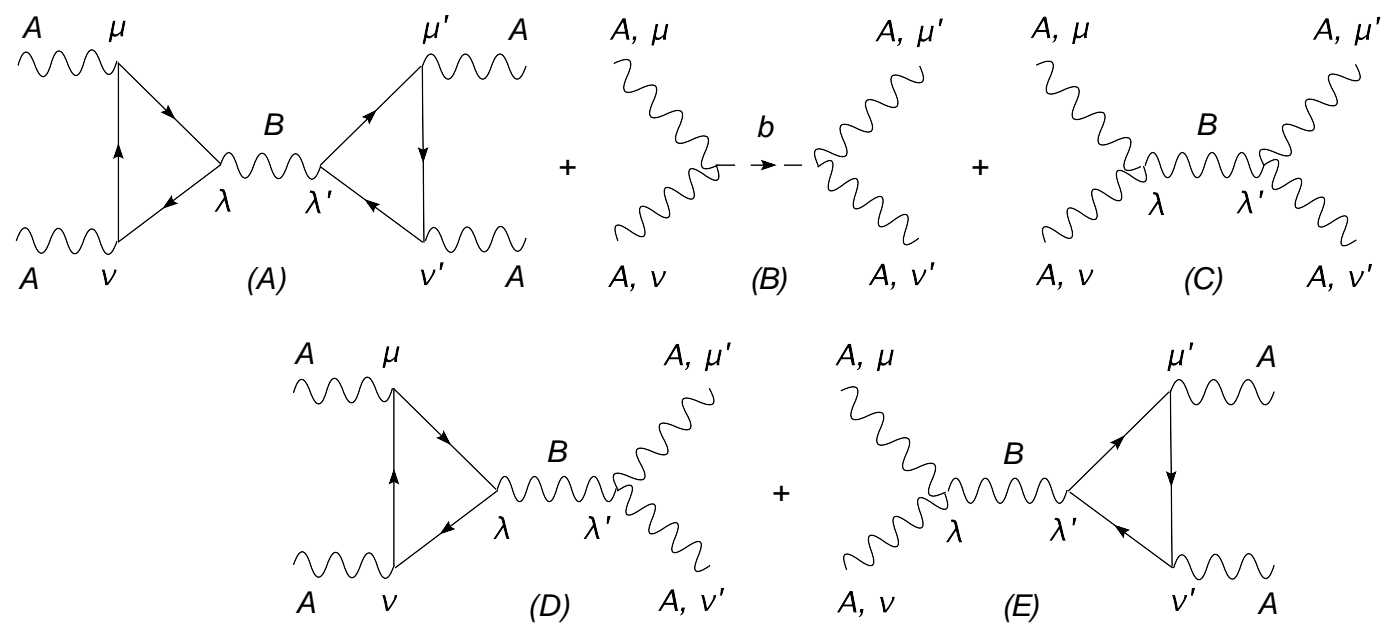

Figure 4: Diagrams involved in the unitarity analysis with CS interactions.

while the $B B B$ triangle is shift-independent as a result of Bose symmetry. In the equation above the distribution of the partial anomalies is such that $a_{1}=a_{2}$ and $a_{1}+a_{2}+a_{3}=a_{n}$ with $a_{n}=i /(2 \pi)^{2}$ being the total anomaly.

It can be shown that $\beta$ in (12) can be fixed by the Ward identity on $A$, giving a conserved vector current (CVC), or can be left arbitrary at the price of introducing a CS interaction. Both approaches give, though, the same physical result. In our parametrization the CVC condition that fixes the two divergent amplitudes of the anomaly diagram is obtained for $\beta=-1 / 2$. As we have already mentioned, in general, external Ward identities could bring these two invariant amplitudes to a paramaterization which differs from Rosenberg's original one [15] and that are typical of a given theory of this type 1 . However, as far as (11) is observed, a diagrammatic cancelation holds, as one can show by a direct computation and as illustrated in Fig. 4. CS interactions, present in Fig. 4, in this case, can be "absorbed" into a standard definition of the (BAA) triangle diagram with a CVC condition on A.

\section{The second window: effective models from string theory (MLSOM and such)}

As we have mentioned, if one of the possible pictures that points toward a modified axion is that of partial decoupling, a second view is related to the higher dimensional origin of the anomaly. This second picture, probably less economical compared to the first, can't be ruled out either and a growing literature [16] on the subject of intersecting brane

\footnotetext{
${ }^{1}$ Notice that different parameterizations, in this case, are not just connected via Schouten's relation.
} 


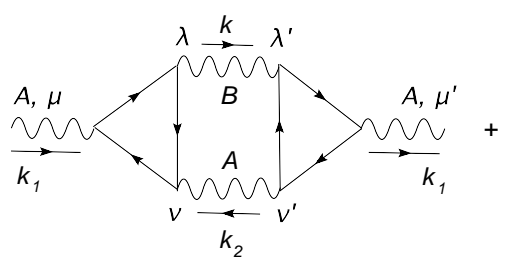

(A)

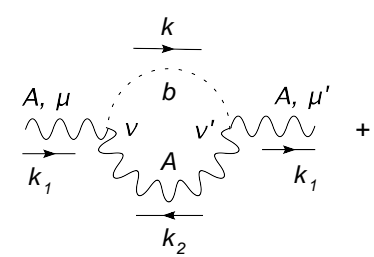

(B)

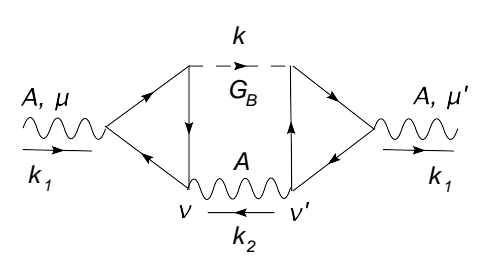

(C)

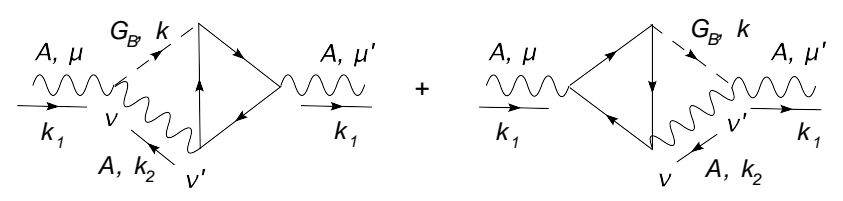

(D)

(E)

Figure 5: Diagrammatics for the cancelation of the gauge dependence in the self-energy of $A$

models testifies of this broad interests. Similar pictures, motivated by the study of various models of anomaly cancelations involving brane scenarios with extra dimensions and an anomaly inflow, have also been formulated [17, 18]. These models are characterized by extended anomalous abelian gauge structures.

Abelian extensions of the SM can be motivated exactly as we have discussed in the introduction. We could easily entertain the idea that a massive axion, whose mass is unspecified, appears in the spectrum. In the $\theta$-vacuum the mass of this axion can be very small and be characterized by an interaction to the fermion sector that is proportional to the fermion mass. If we neglect every additional phase dependent potential - as we lower the energy scale at which we resolve the theory - except for the instanton corrections from the $\theta$-vacuum, the low energy axion develops a mass which is of the same order as the standard Peccei-Quinn axion [19]. One important property to check is the exact masslessness of the left-over symmetry (in our simple example this is the masslessness of A) and the gauge independence of the corresponding corrections, which follow the patterns of cancelation described diagrammatically in Fig. 5. The disappearance of the spurious s-channel poles is obtained by combining the diagrams in a specific pattern dictated by a loop expansion. For instance, the vertex counterterms in this expansion counts one power of $h$, and, as one can check, their sum is unambiguously defined and gauge independent.

We consider the case of an $S U(3)_{C} \times S U(2)_{W} \times U(1)_{Y} \times U(1)_{B}$ model, with an anomalyfree hypercharge (traceless with the ordinary generators of the SM), the SM fermion content and one shifting axion, $b$. We denote with $Y_{B}$ the extra generator of the B gauge boson. The only anomalous contributions are coming from the $Y-Y_{B}$ mixing which 


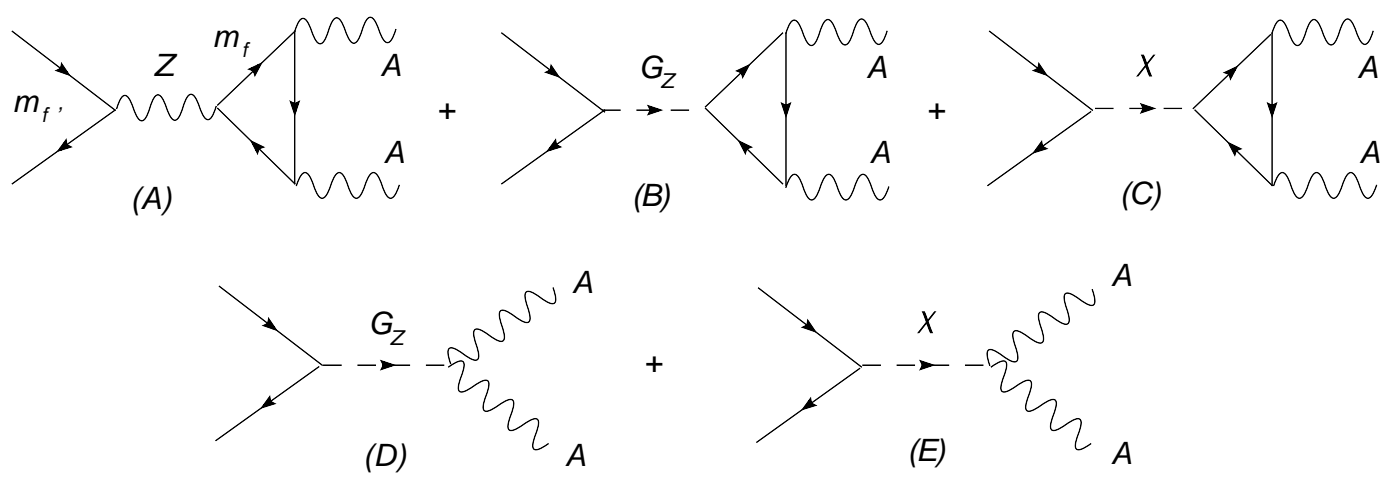

Figure 6: Contributions to two-photon production from $q \bar{q}$ annihilation

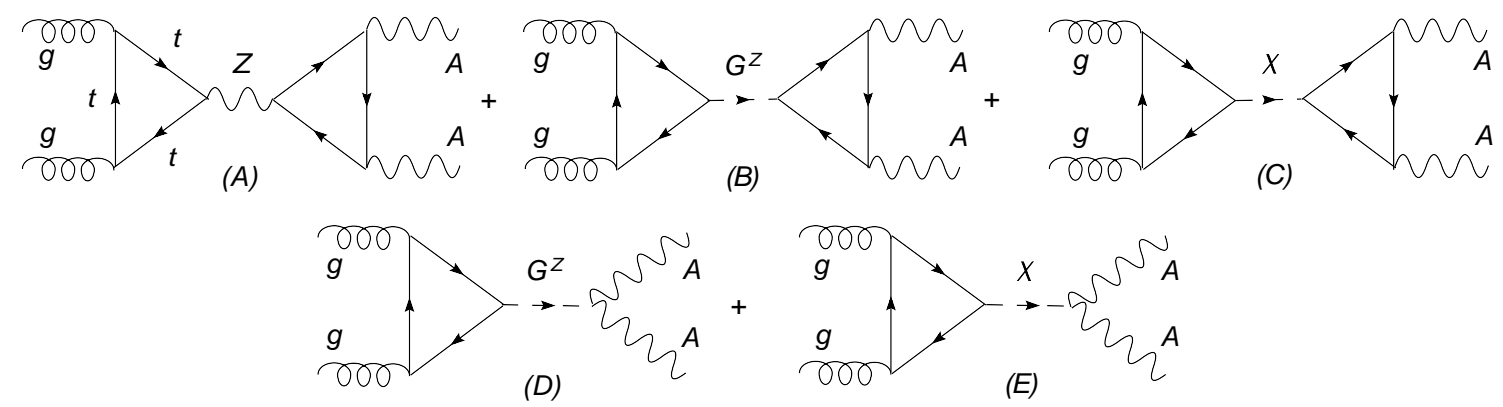

Figure 7: $Z$ exchange from anomalous gluon fusion

are canceled by external Ward identities. Also in this case direct CS interactions are absent. In this case, as in the previous case, we have already shown that the axion must be rotated in the broken phase and expressed in terms of a physical axi-Higgs and two Nambu-Goldstone modes

$$
b=c \chi+c_{1} G_{1}^{0}+c_{2} G_{2}^{0},
$$

where $c$ and $c_{i}$ are dimensionless, computable but model dependent coefficients. At a second stage, the two goldstones can be expressed in terms of $G_{Z}$ and $G_{Z^{\prime}}$, the corresponding goldstones of the two neutral massive gauge bosons.

\subsection{The axion and anomaly effects at the LHC}

Moving our analysis toward possible discoveries of anomaly effects at the LHC, here we briefly discuss the role played by the axi-Higgs and its mixing in simple partonic processes. 

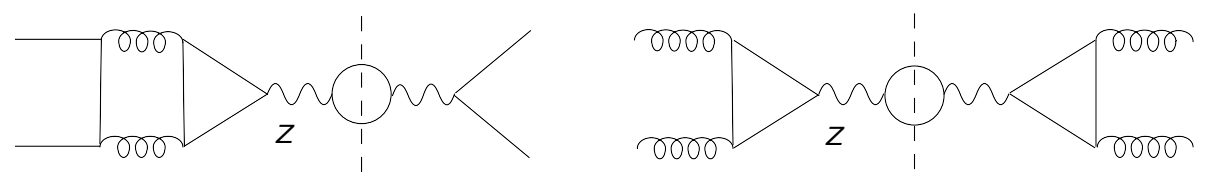

Figure 8: $\mathrm{O}\left(\alpha_{s}^{2}\right)(\mathrm{NNLO})$ corrections in an anomalous Drell-Yan cross section

We have selected one of the simplest processes that can be studied in this new framework, which is the $Z \gamma \gamma$ vertex. Also in this case, a detailed unitarity analysis of the model can be pursued but details will be given elsewhere. We show in Figs. 6 and 7 the set of contributing diagrams that are responsible for an anomalous decay of the $Z$ gauge boson produced from a quark-antiquark collision. The decay channel shown here is the 2-photon decay. Similar processes involving gluons could be taken into consideration. The process occurs with a virtual $Z$ and two real photons, while for an on-shell $Z$ it can take place with one direct and one resolved photon in the final state. A similar decay channel may involve gluons or be mediated by the only $Z^{\prime}$ gauge boson of this extension. This is generated by the mixing of $Y, Y_{B}$ with the neutral component of $S U(2)$. The role played by the triangle anomaly in these type of processes is quite subtle, and is related both to massless and to massive fermion effects, as opposed to the SM, where the anomalous contributions are due only to the difference in mass of each generation of fermions.

In order to simplify the treatment, it is convenient to introduce some notation. We define the chiral asymmetries for the traces of the generators $X_{i}$, with $i$ any latin index labeling both the abelian and the non-abelian cases. All the fermions (f) on which we sum are assumed to be in the same generations of the SM and we will neglect the sum over different generations just for simplicity. These asymmetries are given by the differences between the left (L) and (R) chiral components for each fermion

$$
\theta_{X Y Z}^{(S M) f}=\operatorname{Tr}_{f}\left(X_{i}^{L} X_{j}^{L} X_{k}^{L}\right)_{S M}-\operatorname{Tr}_{f}\left(X_{i}^{R} X_{j}^{R} X_{k}^{R}\right)_{S M} .
$$

We adopt a similar definition for generators with charges in the extended model (an example is the MLSOM of [1])

$$
\theta_{X Y Z}^{(M L S O M) f}=\operatorname{Tr}_{f}\left(X_{i}^{L} X_{j}^{L} X_{k}^{L}\right)_{M L S O M}-\operatorname{Tr}_{f}\left(X_{i}^{R} X_{j}^{R} X_{k}^{R}\right)_{M L S O M},
$$

the simplest of them having a single anomalous $U(1)$, (denoted as $U(1)_{B}$, beside the hypercharge. In this case, the anomalous contributions involve the $B W W$ and the $B Y Y$ triangles, where $Y$ is the hypercharge, which contribute with non vanishing traces, while for the remaining contributions we have the usual conditions

$$
\sum_{f} \theta_{X_{i} X_{j} X_{k}}^{(S M) f}=0
$$


which hold only in the case of the generators of the SM. The derivation of the final expression is rather involved, however the crucial step in the derivation is to separate the massive $\left(\Delta\left(m_{f}\right)\right)$ from the massless $(\Delta(0))$ contributions of the anomalous diagram $\Delta^{\lambda \mu \nu}\left(m_{f} \neq 0\right)$ as

$$
\Delta^{\lambda \mu \nu}\left(m_{f}\right)=\Delta^{\lambda \mu \nu}\left(m_{f} \neq 0\right)-\Delta^{\lambda \mu \nu}\left(m_{f}=0\right)
$$

and by defining $\Delta^{\lambda \mu \nu}(0) \equiv \Delta^{\lambda \mu \nu}\left(m_{f}=0\right)$, after some manipulations we are left with the following expression of the $Z \gamma \gamma$ amplitude in the physical basis in the broken (HiggsStückelberg) phase

$$
\begin{aligned}
A_{Z \gamma \gamma}^{\lambda \mu \nu}=\underline{\Delta}^{\lambda \mu \nu}\left(m_{f}\right) & +\underline{\Delta}^{\lambda \mu \nu}(0) \\
=\underline{\Delta}^{\lambda \mu \nu}\left(m_{f}\right) & +\frac{1}{4} \sum_{f}\left[Q_{B}^{L} Q_{Y}^{L 2}-Q_{B}^{R} Q_{Y}^{R 2}\right] \Delta_{A V V}^{\lambda \mu \nu}(0) R^{B Y Y} \\
& +\frac{1}{4} \sum_{f} Q_{B}^{L f}\left(T_{L}^{3 f}\right)^{2} \Delta_{A V V}^{\lambda \mu \nu}(0) R^{B W W}
\end{aligned}
$$

where

$$
\begin{aligned}
R^{B Y Y} & =\left(O_{Y \gamma}^{A}\right)^{2}\left(O^{A}\right)_{B Z} \\
R^{B W W} & =\left(O_{W \gamma}^{A}\right)^{2}\left(O^{A}\right)_{B Z}
\end{aligned}
$$

describe the rotation from the interaction to the mass eigenstates of the model [1]. The massive contributions $\triangleq\left(m_{f}\right)$ contain suitable combinations of all the generators, while $\Delta(0)$ is the new massless part of the amplitude which is absent in non-anomalous abelian interactions. This second contribution can be given in terms of standard AVV diagrams, as shown in 18, having absorbed some generalized Chern-Simons terms in the amplitude. These two amplitudes both satisfy the Landau-Yang theorem for the on-shell decay of the $Z$ into two massless photons, which has to vanish. The analysis, here shown for a single $U(1)$, can be extended to models with more anomalous $\mathrm{U}(1)$ 's and shifting axions, following a similar approach, along the lines of [1].

\subsection{A brief unitarity analysis}

It is interesting to analize the structure of the various contributions which involve these anomalous vertices in the case of neutral currents. Once more we reconsider Fig. 6. We start from the annihilation of a quark antiquark pair in the parton model. Notice that, for light quarks, the contributions in Fig. 6 simplify, since the goldstones and the axi-Higgs decouple from the fermions (for the sake of clarity, we have included a mass $m_{f}$ on each diagram to emphasize the presence of a mass dependent coupling). In this case the only 
contribution in the quark annihilation channel is due to diagram (A). Similar anomalous channels, related to $Z$ production, are shown instead in Fig. 7. The production of an axi-Higgs also takes place mediated by fermions in the initial state, similarly to the Higgs. We remark that the coupling of the axi-Higgs to the fermions is similar to the Higgs coupling. Corrections to the SM rates of similar processes are due both to the structure of the anomaly traces in each AAA or AVV (Axial or Vector triangle diagram), to the mass differences of the fermions in a given generation, and to the presence of direct WessZumino interactions, which are however suppressed by powers of $1 / M$. For instance, a process characterized by direct production via gluon fusion $g g \rightarrow Z$ not mediated by a heavy fermion in the initial state would similarly be suppressed by the same factor. Analysis of this type can be carried out in a systematic way.

\section{3 "Anomalous" Drell Yan at NNLO}

A final comment concerns the possibility of detecting new effects in neutral current exchanges in the Drell-Yan process. The simplest example amounts to an anomalous contribution that starts at order $\alpha_{s}^{2}$, which is clearly neglected in typical SM computations, due to the vanishing anomaly traces. We show the two relevant diagrams in Fig. 8. They correspond to the interference of the two loop $q \bar{q}$ annihilation into a lepton pair with the analogous channel at tree-level, and the interference of the two gluon fusion diagrams, which is also of the same order. In this case, most of the NNLO QCD analysis performed in the last few years will be crucial to achieve sufficient precision on the $\mathrm{Z}$ resonance in order to extract some essential information on this anomalous process.

\section{Conclusions}

We have illustrated some of the physical consequences that are inevitable when an anomalous abelian gauge interaction appears at low energy. The presence of a new particle, the axi-Higgs, whose mass is the result both of electroweak symmetry breaking and of the phase dependent potential(s) generated in the process of breaking of a symmetry at higher energy, shows a direct gauge interaction of PQ type with some specific features that are clearly missing from the Standard Model but also in the case of the traditional PQ axion model. We have argued that the effective interaction of the axi-Higgs to the gauge bosons can be generated, apart from a variety of geometrical constructions performed in the context of string theory or by anomaly inflow mechanisms from extra dimensions, in a more economical way by simply integrating out some heavy degrees of freedom (Higgs/fermions) of an anomaly-free theory at a higher energy. The model can be realized by two Higgs 
fields, one heavy and one light and an anomaly-free $U(1)$ extra gauge interaction, broken by the heavier Higgs at a higher scale. The same scale is responsible for the suppression of the interaction of the pseudoscalar with the gauge fields at low energy. The mass of the pseudoscalar and its gauge interactions are, therefore, unrelated. This construction renders the Wess-Zumino term simply a low-energy manifestation of incomplete decoupling of a heavier Higgs. Analysis of decoupling of a chiral fermions are available from the previous literature [20] and so are the attempts to look at variants of the PQ axion [21], while a heavy axion that mixes with the Higgs has also been considered [22]. On the other hand the Stückelberg mechanism has also received a renewed attention [23]. Here, our attempt has been to combine several of these ideas into a fruitful form and we have discovered a central avenue: the crucial role played by the WZ term, generated by more economical or by more ambituous constructions. We have shown that, in all these cases, the effective theory that is generated is non-renormalizable but built so that gauge invariance and unitarity are preserved. More details of this analysis will be presented in a forthcoming work.

\section{Acknowledgements}

We thank Marco Guzzi and Simone Morelli for collaborating to this analysis. We thank Elias Kiritsis for collaboration on a previous work that has triggered this analysis. We thank P. Anastasopoulos, M. Bianchi, Marco Roncadelli, Theodore Tomaras and Cosmas Zachos for discussions. The work of C.C. was supported (in part) by the European Union through the Marie Curie Research and Training Network "Universenet" (MRTN-CT-2006035863). He thanks the Physics Department at the University of Crete and in particular Theodore Tomaras for the kind hospitality. N.I. was partially supported by the Research Program PYTHAGORAS II of the Greek Ministry of Education.

\section{References}

[1] C. Corianò, N. Irges and E. Kiritsis, "On the effective theory of low scale orientifold string vacua", Nucl.Phys. B746 (2006), 77.

[2] S.A. Abel, J Jaeckel, V.K. Khoze and A. Ringwald, "Illuminating the string sector of string theory by shining light through a magnetic field "hep-ph/0608248; A. Ringwald, "Particle interpretation of the PVLAS data", arXiv:0704.3195[hep-ph].

[3] A. Mirizzi, G. Raffeldt and P.D. Serpico, "Signatures of axion-like particles in the spectra of TeV gamma-ray sources" arXiv:0704.3044[hep-ph]. 
[4] F. Wilczek, "Problem of strong P and T invariance in the presence of Instanntons", Phys. Rev. Lett.40, 279 (1978).

[5] P. Sikivie, "Axion searches", Nucl.Phys.Proc.Suppl.87, (2000) 41,hep-ph/0002154

[6] C. Corianò and N. Irges," Folding Froggatt-Nielsen into the Stueckelberg-Higgs mechanism in anomalous U(1) models", presented at HEP2006, Recent Developments in High Energy Physics and Cosmology, The Annual Workshop of the Hellenic Society for the study of High Energy Physics, Ioannina, Greece, April 13-16, 2006, hep-ph/0612128.

[7] D. J. Gross and R. Jackiw, "Effect of anomalies on quasirenormalizable theories", Phys.Rev.D6 (1972) 477.

[8] L.D. Faddeev and S. L. Shatashvili, "Realization Of The Schwinger Term In The Gauss Law And The Possibility Of Correct Quantization Of A Theory With Anomalies", Phys.Lett. B167 (1986) 225.

[9] J. Preskill, "Gauge anomalies in an effective field theory", Ann. Phys. 210, (1991) 323.

[10] N. V. Krashnikov, " $\gamma_{5}$ anomalies in non-local gauge theories", JETP Lett.41(1985), 586.

[11] C. Bouchiat, J. Iliopoulos, P. Meyer, "An Anomaly Free Version Of Weinberg's Model", Phys.Lett. B38(1972) 519.

[12] P. Anastasopoulos, M. Bianchi, E. Dudas, E. Kiritsis, "Anomalies, anomalous U(1)'s and generalized Chern-Simons terms", JHEP 0611, (2006) 057.

[13] PVLAS Collaboration (E. Zavattini et al.), "PVLAS: Probing vacuum with polarized light" Presented at QCD 05: 12th International QCD Conference: 20 Years of the QCD-Montpellier Conference, Montpellier, France, 4-8 Jul 2005, hep-ex/0512022

[14] E. Masso, J. Redondo, "Evading astrophysical constraints on axion-like particles", JCAP 0509, (2005) 015.

[15] L. Rosenberg, Phys. Rev. 129, 2786, 1963.

[16] E. Kiritsis, "D-branes in standard model building, gravity and cosmology", Fortsch.Phys.52 (2004) 200.

[17] C.T. Hill "Anomalies, Chern-Simons terms and chiral delocalization in extra dimensions", Phys.Rev. D71 (2005) 046002; C. T. Hill and C. K. Zachos, "Dimensional deconstruction and Wess-Zumino-Witten terms", Phys.Rev. D73 (2006) 085001. 
[18] G. von Gersdorff and M. Quiros, "Localized anomalies in orbifold gauge theories", Phys.Rev.D68 (2003) 105002.

[19] R. D. Peccei and H. R. Quinn, "Constraints imposed by CP conservation in the presence of instantons" Phys. Rev. D 16 (1977) 1791.

[20] F. Feruglio, A. Masiero and L. Maiani, "Low-energy effects of heavy chiral fermions" Nucl.Phys.B387, (1992) 523.

[21] S. Chang and J. E. Kim, "Is heavy quark axion necessarily hadronic axion?" Phys.Rev.D49 (1994) 2161.

[22] V. A. Rubakov, "Grand unification and heavy axion", JETP. Lett. 65 (1997) p. 621 hep-ph/9703409.

[23] B. Kors and P. Nath, "Aspects of the Stückelberg extension", JHEP 0507 (2005) 069. 